10-1-1996

\title{
An Exactly Soluble Fresnel Diffraction Model of Two-Slit Interference
}

James A. Lock

Cleveland State University, j.lock@csuohio.edu

Follow this and additional works at: https://engagedscholarship.csuohio.edu/sciphysics_facpub

Part of the Physics Commons

How does access to this work benefit you? Let us know!

\section{Publisher's Statement}

Copyright 1996 American Association of Physics Teachers. The article appeared in American Journal of Physics 64 (1996): 1307-1311 and may be found at http://aapt.scitation.org/doi/pdf/ 10.1119/1.18375

\section{Original Citation}

Lock, James A. "An Exactly Soluble Fresnel Diffraction Model of Two-Slit Interference." American Journal of Physics 64 (1996): 1307-1311.

\section{Repository Citation}

Lock, James A., "An Exactly Soluble Fresnel Diffraction Model of Two-Slit Interference" (1996). Physics Faculty Publications. 102.

https://engagedscholarship.csuohio.edu/sciphysics_facpub/102

This Article is brought to you for free and open access by the Physics Department at EngagedScholarship@CSU. It has been accepted for inclusion in Physics Faculty Publications by an authorized administrator of EngagedScholarship@CSU. For more information, please contact library.es@csuohio.edu. 


\title{
An exactly soluble Fresnel diffraction model of two-slit interference
}

\author{
James A. Lock
}

\section{INTRODUCTION}

Many physics majors, though able to perform complicated mathematical calculations, seem to have trouble understanding what the mathematics represents in physical terms. Thus one of the challenges in teaching undergraduate courses for physics majors is to take formulas and expressions that are relatively mathematically complicated and explain how the various terms in the expressions illustrate the physical principles involved in the phenomenon under study. One of the most useful aids in this regard is the occasional physical situation that has the virtues of being both mathematically exactly soluble and rich in physical content. In these situations, issues of mathematical complexity no longer occur at the end of the calculation and we are left with a clear and unimpeded view of the physics that is being studied.

When teaching a course on optics. Fresnel diffraction poses such a challenge. On the one hand, it is a physically important phenomenon since it is the bridge connecting the seemingly disparate topics of geometrical ray optics and wave interference. By analogy, it also helps students understand the connection between the complementary worlds of classical particle trajectories and the superposition of quantum mechanical matter waves. But on the other hand, Fresnel diffraction is mathematically complicated by the fact that even for simple apertures and obstacles, the resulting diffraction integrals are not exactly soluble. As a result, we rely on a number of graphical techniques such as Cornu's spiral ${ }^{1.2}$ and Fresnel zone plates, ${ }^{3}$ and numerical recipes for computing auxiliary Fresnel functions $s^{4.5}$ and Lommel functions ${ }^{6-8}$ in order to produce sketches or graphs of the light intensity as a function of position which assist us in understanding the physics that underlies the mathematics of Fresnel diffraction.
In this paper I examine an exactly soluble version of Young's two-slit interference problem in the context of Fresnel diffraction in the hope that it may be of use to others who teach undergraduate courses in optics. I consider the diffraction and interference of two initially widely separated parallel Gaussian light beams propagating in the $z$ direction. In the $z=0$ plane, each beam has an electric field half-width $w_{0}$. The centers of the beams are separated by the distance $b \gg 2 w_{0}$. This situation models a plane wave normally incident on two Gaussian apertures in the $z=0$ plane. The resulting diffraction plus interference pattern is qualitatively illustrated ${ }^{9}$ in Fig. 1. A viewing screen placed near the aperture plane at small $z>0$ shows two bright spots of light separated by the distance $b$ which are easily understood in terms of the propagation of geometrical light rays through the two apertures. For larger $z$ the diffraction pattern for each of the two apertures begins to emerge. For yet larger $z$, the two diffraction patterns partially overlap producing an interference pattern in the overlap region. In the far-zone limit, the overlap of the two diffraction patterns is nearly complete and the resulting interference pattern is the familiar Young twoslit interference result.

The far-zone Fraunhofer limit of this phenomenon is treated in elementary level textbooks. ${ }^{10}$ The transition region, though occasionally qualitatively described, ${ }^{9}$ is seldom quantitatively examined due to the mathematical complexity of the Fresnel integrals involved. ${ }^{11}$ Our version of the twoslit problem bypasses these difficulties, permitting a quantitative understanding of the transition region. Our solution to this problem proceeds as follows. In Sec. II we briefly summarize Fresnel diffraction of a single Gaussian beam. In Sec. 


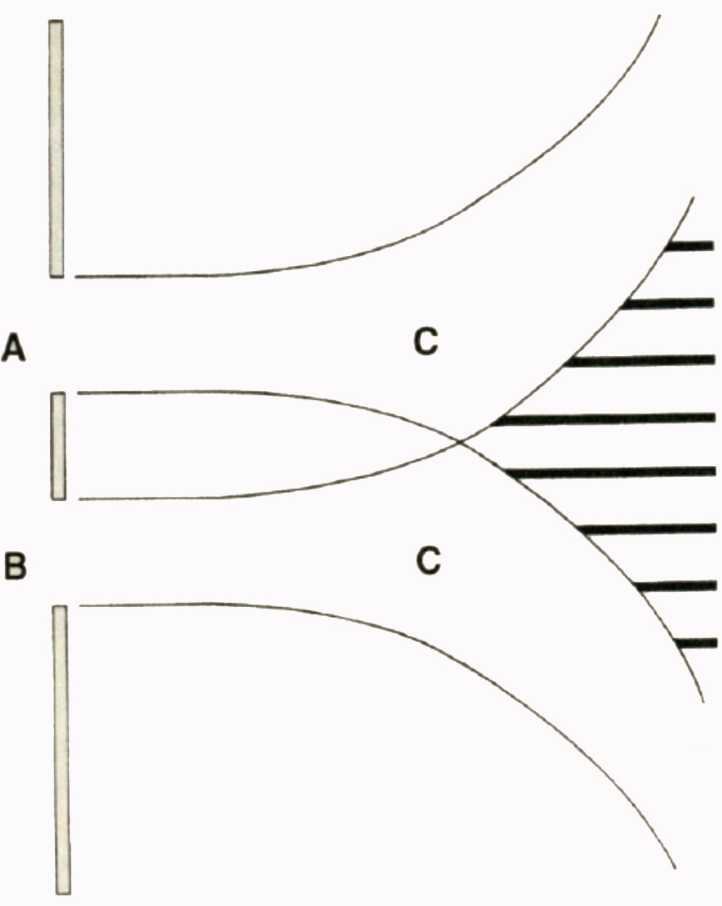

Fig. 1. The two-slit interference geometry. Normally incident light passes through two apertures $\mathrm{A}$ and $\mathrm{B}$. It diffracts in region $\mathrm{C}$. The two diffraction patterns overlap, forming an interference pattern in region $\mathrm{D}$.

III we examine the superposition of two such parallel beams which are initially widely separated. Our final conclusions are presented in Sec. IV.

\section{PROPAGATION OF A GAUSSIAN BEAM}

The equations describing the paraxial propagation of a Gaussian laser beam appear in many books. ${ }^{12-14}$ Since these equations are necessary for an understanding of the results of Sec. III, they are briefly summarized here. Consider a monochromatic light beam of wave number $k$ and angular frequency $\omega$ propagating in the $z$ direction. The electric field of the beam in the $z=0$ plane is given by $\mathbf{E}\left(x^{\prime}, y^{\prime}, 0, t\right)$. The Fresnel diffracted beam for $z>0$ is then

$$
\begin{array}{r}
\mathbf{E}(x, y, z, t)=\frac{-i k}{2 \pi z} \int_{-\infty}^{\infty} d x^{\prime} \int_{-\infty}^{\infty} d y^{\prime} \mathbf{E}\left(x^{\prime}, y^{\prime}, 0, t\right) \\
\times e^{i k z} e^{i(k / 2 z)\left(x-x^{\prime}\right)^{2}} e^{(i k / 2 z)\left(y-y^{\prime}\right)^{2}} .
\end{array}
$$

If the beam profile in the $z=0$ plane is Gaussian, i.e.,

$$
\mathbf{E}\left(x^{\prime}, y^{\prime}, 0, t\right)=E_{0} e^{-\left(x^{\prime 2}+y^{\prime 2}\right) / w_{0}^{2}} e^{-i \omega t} \hat{u}_{x},
$$

then Eq. (1) is exactly integrable giving

$$
\mathbf{E}(x, y, z, t)=\frac{E_{0}}{D} e^{-\left(x^{2}+y^{2}\right) / D w_{0}^{2}} e^{i k z-i \omega t} \hat{u}_{x},
$$

where

$$
D=1+\frac{2 i z}{k w_{0}^{2}} .
$$

Similar equations describe the magnetic field of the beam. This beam is not an exact solution of Maxwell's equations since the induced components of the $\mathbf{E}$ and $\mathbf{B}$ fields are absent. ${ }^{15,16}$ But if the beam is not tightly focused in the $z=0$ plane, i.e., $w_{0} \gg \lambda$, the induced fields are negligible and Eqs. (3) and (4) are a good approximation to the propagating beam.

The light intensity of the beam is

$$
I(x, y, z)=\frac{\mathbf{E}^{*} \cdot \mathbf{E}}{2 \mu_{0} c}=\frac{I_{0} w_{0}^{2}}{w^{2}(z)} e^{-2\left(x^{2}+y^{2}\right) / w^{2}(z)},
$$

where

$$
I_{0}=\frac{E_{0}^{2}}{2 \mu_{0} c}
$$

and

$$
w(z)=w_{0}\left(1+\frac{4 z^{2}}{k^{2} w_{0}^{4}}\right)^{1 / 2} .
$$

In the far zone where $2 z / k w_{0}^{2} \gg 1$, the beam intensity becomes

$$
\lim _{z \rightarrow \infty} I(x, y, z)=\frac{k^{2} w_{0}^{4} I_{0}}{4 z^{2}} e^{-k^{2} w_{0}^{2} \theta^{2} / 2},
$$

where

$$
\theta \approx \frac{\left(x^{2}+y^{2}\right)^{1 / 2}}{z}
$$

is the angle between the field point $(x, y, z)$ and the $z$ axis

The various terms in Eqs. (2)-(8) have the following physical interpretations. In the $z=0$ plane the electric field half-width of the beam is $w_{0}$. For $z>0$ the beam profile remains Gaussian, but the beam diffractively spreads, having the electric field half-width $w(z)>w_{0}$. In the far-zone limit $2 z / k w_{0}^{2} \gg 1$, the angular half-width of the electric field is $2 / k w_{0}$. In Eq. (5), as the beam diffractively spreads, the peak value of the intensity $I_{0} W_{0}^{2} / w^{2}(z)$ decreases, consistent with conservation of energy for all values of $z$. In the $z=0$ plane, the surface of constant phase of the electric field in Eq. (2) is flat. As the beam spreads, the surfaces of constant phase become curved and are described in Eq. (3) by the exponential of the imaginary part of $-\left(x^{2}+y^{2}\right) / D w_{0}^{2}$. For $2 z / k w_{0}^{2} \gg 1$ the shape of the wave front becomes spherical and has radius

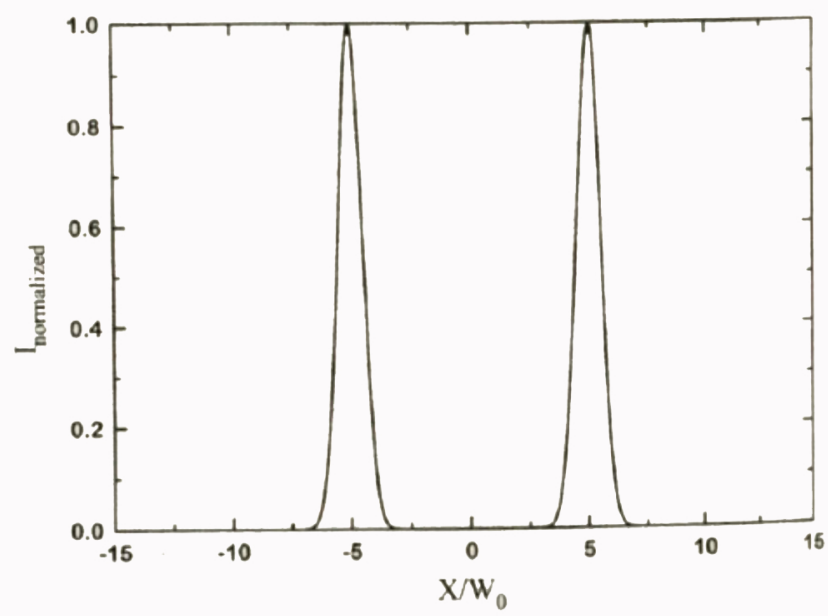

Fig. 2. Intensity as a function of position in the $\boldsymbol{u}=0$ plane for two Gaussian beams having a spacing-to-width ratio $b / w_{0}=10.0$. 

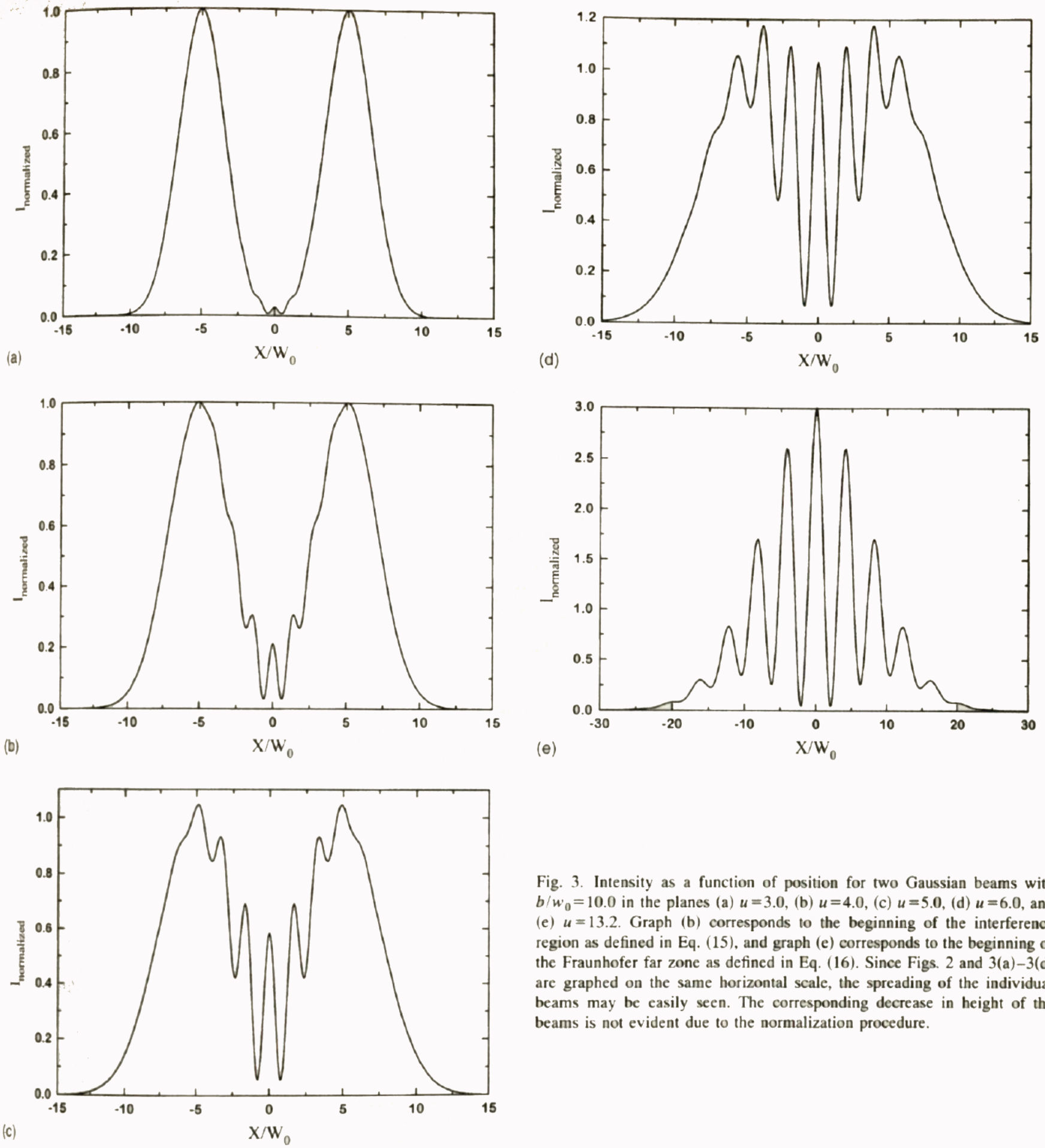

Fig. 3. Intensity as a function of position for two Gaussian beams with $b / w_{0}=10.0$ in the planes (a) $u=3.0$, (b) $u=4.0$, (c) $u=5.0$, (d) $u=6.0$, and (e) $u=13.2$. Graph (b) corresponds to the beginning of the interference region as defined in Eq. (15), and graph (e) corresponds to the beginning of the Fraunhofer far zone as defined in Eq. (16). Since Figs. 2 and 3(a)-3(d) are graphed on the same horizontal scale, the spreading of the individual beams may be easily seen. The corresponding decrease in height of the beams is not evident due to the normalization procedure.

z. Thus, in this limit, the beam propagates as an outgoing spherical wave from an effective point source located at the center of the beam focal waist.

The realm of geometrical ray optics for this Gaussian beam ends at the value of $z$ where the diffractive spreading of the beam becomes appreciable. This point is commonly taken to be the end of the beam focal waist where

$$
u \equiv \frac{2 z}{k w_{0}^{2}}=1.0 .
$$

Similarly, the realm of Fraunhofer diffraction can be defined to begin at the value of $z$ where the diffractive spreading of

the beam given by Eq. (7) deviates from being a function of $\theta$ alone by $1 \%$. This occurs for $u \approx 7.0$.

TWO PARALLEL GAUSSIAN BEAMS AS A MODEL OF YOUNG'S TWO-SLIT INTERFERENCE PROBLEM

We now consider two parallel Gaussian beams each of electric field half-width $w_{0}$ separated by the distance $b \gg 2 w_{0}$ along the $x$ axis in the $z=0$ plane. Using Eq. (3), the total electric field for $z>0$ is 


$$
\begin{aligned}
\mathbf{E}(x, y, z, t)= & \frac{E_{0}}{D} e^{i k z-i \omega t}\left(e^{-\left[(x-b / 2)^{2}+y^{2}\right] / D w_{0}^{2}}\right. \\
& +e^{\left.-\left[(x+b / 2)^{2}+y^{2}\right] / D w_{0}^{2}\right)} \hat{u}_{x} .
\end{aligned}
$$

After a reasonable amount of algebra, the two-beam intensity normalized to the maximum intensity of each beam at the same value of $z$ is

$$
\begin{aligned}
I_{\text {normalized }}(x, y, z) \equiv & \frac{I_{\text {total }}(x, y, z)}{I_{\text {each }}(\max , z)} \\
= & e^{-2\left[(x-b / 2)^{2}+y^{2}\right] / w^{2}(z)} \\
& +e^{-2\left[(x+b / 2)^{2}+y^{2}\right] / w^{2}(z)} \\
& +2 e^{-b^{2} / 2 w^{2}(z)} e^{-2\left(x^{2}+y^{2}\right) / w^{2}(z)} \\
& \times \cos \left(\frac{2 b u x}{w^{2}(z)}\right) .
\end{aligned}
$$

After collecting together all the common factors and rearranging, Eq. (12) may be rewritten as

$$
\begin{aligned}
I_{\text {normalized }}(x, y, z)= & 4 e^{-b^{2} / 2 w^{2}(z)} e^{-2\left(x^{2}+y^{2}\right) / w^{2}(z)} \\
& \times\left[\cos ^{2}\left(\frac{b u x}{w^{2}(z)}\right)+\sinh ^{2}\left(\frac{b x}{w^{2}(z)}\right)\right] .
\end{aligned}
$$

Equations (12) and (13) are the solution to our version of Young's two-slit interference problem for an arbitrary value of $z$. They possess the virtue of being both mathematically simple and easily interpretable. Equation (12) describes in a natural way the region where the two beams first begin to overlap and interfere. The reason for this is that Eq. (12) emphasizes the independent existence of the two individual beams, i.e., the first two terms on the right-hand side of the equation. The last term in the equation is the interference between the two beams in the region of overlap. The cosine interference factor is spatially modulated by a Gaussian envelope centered on the $z$ axis and having the same width as each of the two individual beams. The peak value of the Gaussian modulation envelope is $2 \exp \left[-b^{2} / 2 w^{2}(z)\right]$. Since the two beams are widely separated in the $z=0$ plane (i.e., $b \gg 2 w_{0}$ ) the peak value of the interference term is negligible for small $z$. As the beams diffractively spread and $w(z)$ increases, the region of overlap increases and the peak value of the interference term correspondingly increases.

As $z$ increases further, the two beams spread to such an extent that they almost completely overlap. The interference pattern then extends over almost the entire profile of each beam. This situation is more naturally described by Eq. (13) in which the interference structure, rather than the independent existence of the two individual beams, is emphasized. The sinusoidal interference factor is again spatially modulated by the Gaussian envelope having the peak value $4 \exp \left[-b^{2} / 2 w^{2}(z)\right]$ which approaches 4.0 as $z \rightarrow \infty$. This limiting value of the peak intensity reflects the fact that each of the normalized beams has a maximum electric field amplitude of unity and constructively interferes on the $z$ axis. The $\sinh ^{2}\left[b x / w^{2}(z)\right]$ term is the remnant of the not quite complete overlap of the two individual beams and acts as a baseline upon which the interference pattern is added. This term approaches zero as $z \rightarrow \infty$ where the two beams completely overlap. In the far-zone Fraunhofer limit, Eq. (13) becomes

$$
\lim _{z \rightarrow \infty} I_{\text {normalized }}(x, y, z)=4 e^{-k^{2} w_{0}^{2} \theta^{2} / 2} \cos ^{2}\left(\frac{k b x}{2 z}\right)
$$

which agrees with the standard Young two-slit interference result, i.e., a sinusoidal interference pattern spatially modulated by the intensity pattern of one of the apertures. ${ }^{17}$

For this version of the two-slit interference problem, the beginning of the interference region and the beginning of the Fraunhofer far-zone region do not depend solely on $u$ as was the case for a single beam in Sec. II. This is because the overlap of the two beams depends both on the rate at which each beam diffractively spreads and on the initial beam separation. The region of beam interference can be defined to begin when the largest value of the interference term in Eq. (12) is about $10 \%$ of the individual peak beam intensities. thereby becoming a non-negligible feature in the overall intensity pattern. This occurs when

$$
u \approx\left[\frac{1}{6}\left(\frac{b}{w_{0}}\right)^{2}-1\right]^{1 / 2}
$$

The beginning of the Fraunhofer far zone can be defined to begin when the peak value of the interference factor in $\mathrm{Eq}$ (13) is about 3.0 which is $75 \%$ of its value in the far zone This occurs when

$$
u \approx\left[\frac{7}{4}\left(\frac{b}{w_{0}}\right)^{2}-1\right]^{1 / 2}
$$

and corresponds to the interference structure strongly dominating over the individual beam intensities.

The behavior of Eqs. (12) and (13) are illustrated in Figs. 2 and 3 for $b / w_{0}=10.0$ and for various values of $u$ from the geometrical limit to the Fraunhofer limit. Figures 2 and 3 illustrate both the qualitative features of the transition described in Sec. I and the interpretation of the various terms in Eqs. (12) and (13) given in this section.

\section{CONCLUSION}

In any advanced physics course, whenever one writes a complicated mathematical expression that describes some phenomenon, one can be sure that each term in the expression has a definite physical interpretation. It is always our goal to find out what those physical interpretations are. This is where the learning of physics occurs. The situations that we analyze in the classroom quite often fall into one of two categories. First, they may be physically realistic but analytically intractable. We can still solve these problems numerically and learn much from them. But in these cases it is sometimes difficult to obtain an intuition of what will happen if we change some of the parameters in the problem without having to resolve it and see what happens. Second, some situations are easy to solve exactly. But these problems are often not very instructive since they are nearly devoid of physical content because of the degree to which they have been idealized in order to be able to solve them exactly. Occasionally, however, we find situations for which the equations are exactly soluble and which are rich in physical understanding. These situations are ideal for bringing out the physics that is described by the mathematics. Our version of Young's two-slit interference problem in the context of the Fresnel diffraction is such a situation which clearly and simply tells the story of the transition between geometrical ray optics and wave interference. 
'E. Hecht, Optics (Addison-Wesley, Reading. MA, 1987), 2nd ed., Sec. 10.3.7.

'M. B. Stewart, "Cornu's spiral on a microcomputer." Am. J. Phys. 54. 280-281 (1986).

Ref. 1, Secs. 10.3.1-10.3.5.

D. S. Burch. "Fresnel diffraction by a circular aperture," Am. J. Phys. 53. 255-260 (1985).

M Abramowitz and I. A. Stegun, Handbook of Mathematical Functions National Bureau of Standards, Washington, DC, 1964), Sec. 7.3.

p. M. Rinard, "Large scale diffraction patterns from circular objects," Am. J. Phys. 44, 70-76 (1976).

M. A. Heald, "Computation of Fresnel diffraction." Am. J. Phys. 54. 980-983 (1986).

"E. A. Hovenac, "Fresnel diffraction by spherical ohstacles," Am. J. Phys. 57. 79-84 (1989).

${ }^{9} \mathrm{~A}$ variation on this figure has appeared in Rct. 1. p. 341. as Fig. $9.5 \mathrm{f}$
"'D. Halliday, R. Resnick, and J. Walker, Fundamentals of Physics (Wiley, New York, 1993), 4th ed., Secs. 411.4 and 40.6

"J. A. Lock. "Numerical methods in optics: A course about learning phrsics through computing." Am. J. Phys. 55, 1121-1125 (1987).

R. J. Pressley (Ed.) Handhook of Lasers (C RC, Cleveland, 1971), pp. 422 and 424

${ }^{17}$ A. E. Siegman, Lasers IUniversity Science Books, Mill Valley, CA, 1986). Secs. 16.3 and 17.1 .

${ }^{14} \mathrm{H}$. A. Haus, Waves and Fields in Optoelectronics (Prentice-Hall, Englewood (lifts. NJ. 1984), Sec. 5.1.

"L. W' Davis. "Theory of electromagnetic beams," Phrs. Rev. A 19. $1177-1174,19791$.

${ }^{16}$ J. P. Barton and D. R. Alexander, "Fifth-order corrected electromagnetic ficld components for a fundamental Gaussian beam." J. Appl. Phvs 66 $2800-28012(19 \times 9)$

Ret. 1, Sec. 10.2.2

Post-print standardized by MSL Academic Endeavors, the imprints of the Michael Schwartz Library at Cleveland State University, 2017. 